\title{
Association of Mother's Genetic Sensitivity to the Taste of 6-N-Propylthiouracil (Prop) and Their Children's Dental Caries Status in Nepal
}

\author{
Nilotpol Kashyap ${ }^{1 *}$, Siraj DAA Khan ${ }^{2}$, SJ Das ${ }^{3}$, Brij Kumar ${ }^{4}$, Manjula Das $^{5}$ and Ashwini Dayma ${ }^{6}$ \\ ${ }^{1}$ Department of Pediatric Dentistry, Chitwan Medical College, Nepal \\ ${ }^{2}$ Pediatric Dentist, Faculty of Dentistry, University of Najran, Saudi Arabia \\ ${ }^{3}$ Department of Periodontology, Regional Dental College, Assam \\ ${ }^{4}$ Department of Pediatric Dentistry, Rungrta College of Dental Sciences and Research, Bhillai \\ ${ }^{5}$ Department of Prosthodontics, Silchar Medical College, Nepal \\ ${ }^{6}$ Department of Community Dentistry, UCMS, Nepal
}

*Corresponding author: Nilotpol Kashyap, Prof and HOD Department of Pediatric Dentistry, Chitwan Medical College, Nepal

\begin{abstract}
Introduction: Dental caries is a multifactorial disease and is one of the most prevalent infectious disease that affects mankind. Young children present a unique risk for dental caries as their host-defense systems and bacterial flora are in the process of being developed and also because the newly erupted tooth surfaces are more susceptible to dental caries. Mothers are the persons who generally influence their children via their own food preferences. Hence the mother's taste perception plays an important role in the development of dental caries in their children. The need of this study is to examine the association of mother's taste perception to 6-n-propylthiouracil with caries prevalence in their young children as well as with other caries risk determinants such as mothers and their children's oral hygiene practices.

Material and Method: 180 pair of mothers and their children in the age group of 3 to 6 years of both sexes were selected for the study \& 6-n-propylthiouracil testing is done. A trained and calibrated examiner who did not have any knowledge of the mother's PROP test performed a comprehensive clinical examination of the children to determine the presence or absence of DMFT/dmft.

Results: It is observed that nonstarter mother and children have higher caries prevalence than medium tasters and supertasters. Discussion- Genetic sensitivity to taste is an inherited trait in children from their parents, inheritance from mother being more pronounced.
\end{abstract}

Conclusion: Dental caries is multifactorial. No significant correlation between susceptibility of mother and child to genetic sensitivity exists, and genetic sensitivity is not the only criteria for severity.

Keywords: 6-n-Propylthiouracil; dental caries; dietary habits

\section{Introduction}

Dental caries is a multifactorial disease and is one of the most prevalent infectious disease that affects mankind. The development of dental caries depends on several critical interactions between a susceptible tooth surface, oral bacteria, fermentable carbohydrates and frequency of consumption of sugar [1,2]. Young children present a unique risk for dental caries as their host-defense systems and bacterial flora are in the process of being developed and also because the newly erupted tooth surfaces are more susceptible to dental caries [3]. Parents must also negotiate the dietary transition from bottle feeding to solid food and also take into consideration the child's tastes. Several risk factors such as salivary counts of mutant's streptococci, past carious experience and frequency of sugar intake have been evaluated to identify children at high risk of caries [1,4-7]. However, none of the currently available caries 
screening methods can identify children at high risk of caries quite effectively $[1,8]$.

The role of diet as a direct cause of dental caries has been extensively reported $[1,4,9,10]$. A high intake of sugar has been correlated with a high dental caries in pre-school and school aged children. A high sugar intake reflects a preference for a sweet substance. Genetic sensitivity to taste may be associated with a preference or no preference of some food by children. Sensitivity to taste is an inherited trait in children [1,11]. In 1991 it was found that there is a genetic variation in the ability to taste the bitterness of the chemical 6-n-propylthiouracil (PROP) [1,12]. This variation was found to be associated with food preferences in children. Fox in 1931 found that some individuals perceived chemicals related to PROP as bitter (supertaster) while others could hardly perceive them at all (nonstarter). Those who are PROP super taster are typically supertasters in general and tend to dislike sweet while those who are PROP none tasters like sweet food and prefer strong tasting food products. Mothers are the persons who generally influence their children via their own food preferences. In many households' mothers generally reward their children using sugary snacks which might increase their children's preference for that kind of food. Hence the mother's taste perception plays an important role in the development of dental caries in their children. This study was carries out with following aims and objectives:

\section{Aim}

To find the association between mother's sensitivity to 6-n-propylthiouracil (PROP) and their children's dental caries status

\section{Objectives}

To compare mother's and child's oral hygiene practices with their dental caries status. To determine the taste quality and taste preferences of food products among these study groups.

\section{Materials and Methods}

220 pairs of mother and children were randomly selected from consecutive children who were accompanied by their mothers visiting the Department of Pedodontics and Preventive Dentistry in the institution. Out of these 220 pairs of mother and children 180 pairs fulfilled the inclusion criteria. A single trained and calibrated examiner who did not have any knowledge of the mother's PROP test performed a comprehensive clinical examination of the children to determine the presence or absence of DMFT/dmft. All examinations were performed with a mouth mirror and explorer in a lighted operatory after taking consent from the mother. Demographic information of mothers, their oral hygiene practices, their oral health status and the presence of grandparents in the household was collected by an open-ended questionnaire. A second questionnaire was used to collect data regarding the oral hygiene and feeding practices of the child (e.g. frequency of tooth brushing, intake of sugary food and frequency of intake of sugary food).

\section{Inclusion Criteria}

\section{For children}

a) Age groups of 3 to 6 years.
b) ASA physical status I/II and stable mental condition
c) Should be accompanied by mother.

\section{For mother}

a) Participant cooperation and acceptance of the study programmer.

b) Should be healthy and mentally stable.

c) Should be literate.

\section{Exclusion Criteria}

\section{For children}

a) Should not be less than three years and not over six years

b) Should not have any medical / hereditary conditions.

c) No acute dental diseases.

\section{For mothers}
a) Should not be pregnant
b) Should not suffer from systemic diseases
c) Should not be allergic to 6-n-propylthiouracil (PROP)
d) Should not be under any medications that could alter the taste sensation and affect salivary flow.

\section{Prop testing}

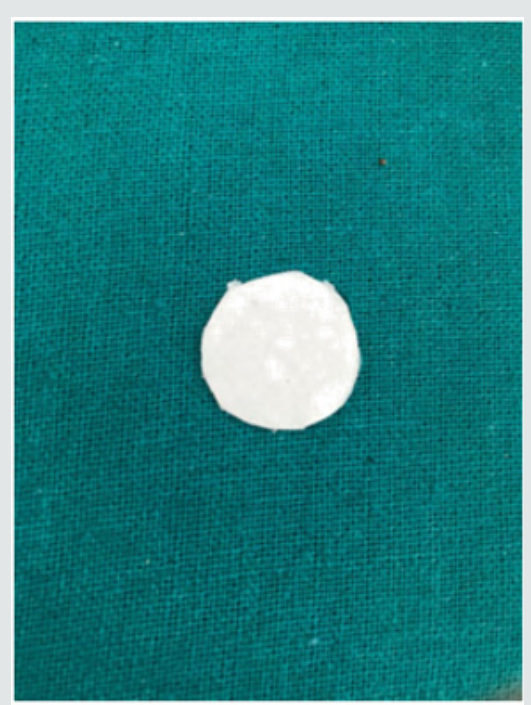

Figure 1: Filter paper with PROP solution.

A single examiner who did not have any knowledge of the mother's and children's oral health status had conducted the PROP test on the mothers. A small piece of filter paper $(2 \mathrm{~cm}$ circle of Whatman's grade 1 filter paper) containing approximately $1.6 \mathrm{mg}$ (measured by calibrated dropper) of 6-n-propylthiouracil (PROP) was used to determine each mother's taste type (Figure 1). The mother was asked to put the piece of filter paper in the mouth and moisten it with saliva for 30 seconds (Figure 2). After removing the filter paper, the mother was asked to quantify the intensity of bitter taste on the modified Green's scale and was classified of supertasters $(>60)$, medium tasters $(12-60)$ and no tasters $(<12)$. 
All examinations and PROP testing were carried out at the same time of the day (mid-morning) throughout the study. All the data gathered was tabulated and statistically analyzed. Student's paired t test and chi square test was done.

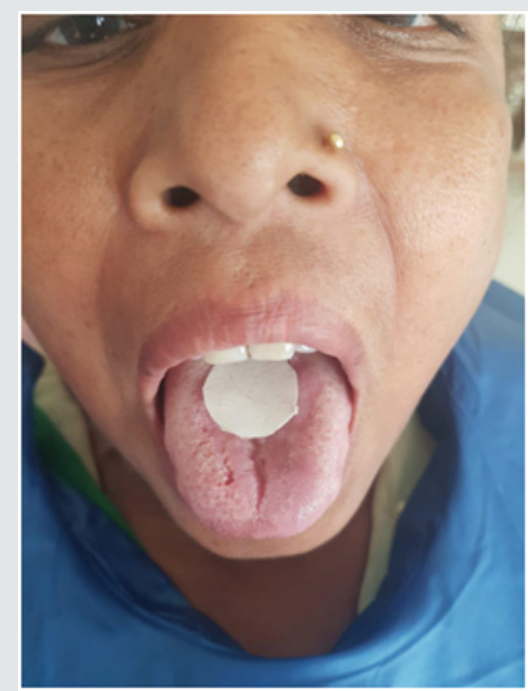

Figure 2: PROP test on mother.

\section{Results}

The sample population consisted of 180 healthy preschool children and their mothers. Children 's ages ranged from three to six years, of which were 68 (37.7\%) boys and were 112 (62.2\%) girls (Figure 3). Mothers of those children varied in age between 21 years and 45 years. Among those mothers, 96 (53.3\%) subjects were PROP non-tasters and $73(40.5 \%)$ subjects were PROP supertasters (Figure 4). 68 (37.78\%) children and 54 (30\%) mothers in this sample reportedly brushed their teeth at least twice a day. 11 (6.11\%) children and 27 (15\%) mothers reportedly never brushed their teeth (Figure 5 and 6). Almost one-third of the children (38.89\%) reportedly consumed given sugary snacks given by grandparents (Figure 7). 58 (79.4\%) children of supertaster mother and $68(87.6 \%)$ supertaster mothers don't like sugary food (p-0.001). On the other hand, 91 (94.7\%) children of non-taster mother and 95 (98.9\%) non-taster mother like sugary food (p0.001) (Figures $8 \& 9$ and Table 1). Caries experience for teeth was evaluated by decayed $(\mathrm{D} / \mathrm{d})$, missing $(\mathrm{M} / \mathrm{m})$, and filled $(\mathrm{F} / \mathrm{f})$ teeth (dmft). The distribution of mean DMFT/dmft for all maxillary and mandibular teeth in both groups is presented in (Figure 10 and Table 2).

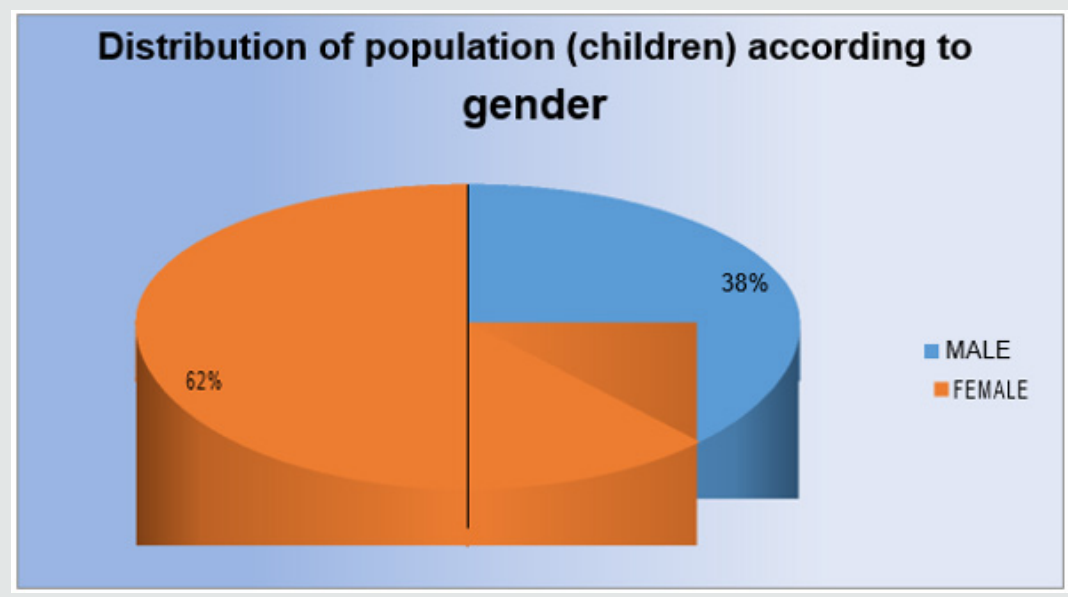

Figure 3: Distribution of population (children) according to gender.

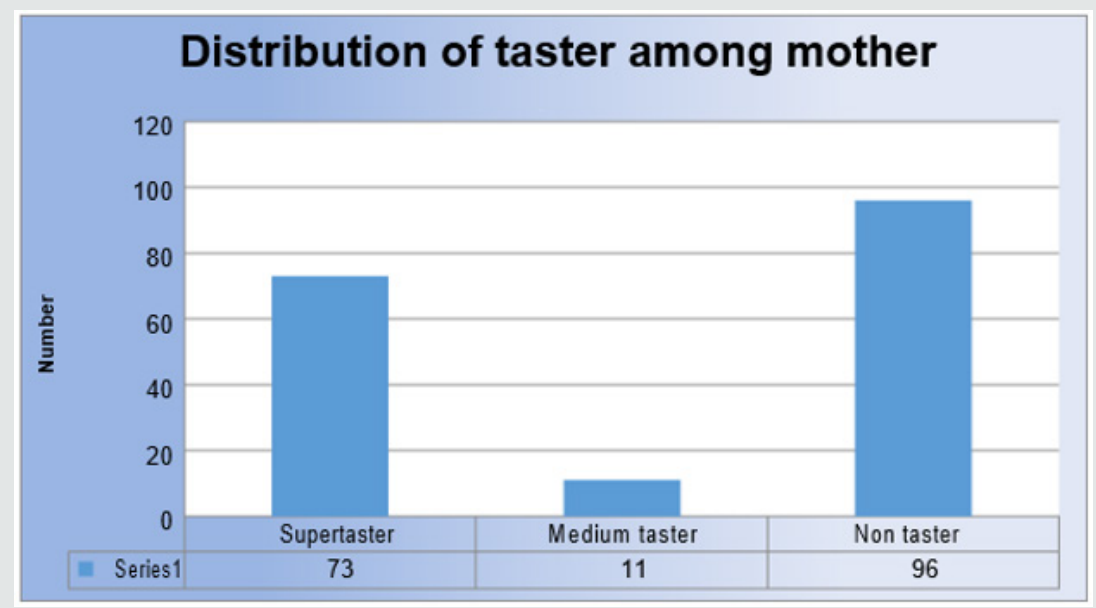

Figure 4: Distribution of taster among mother. 


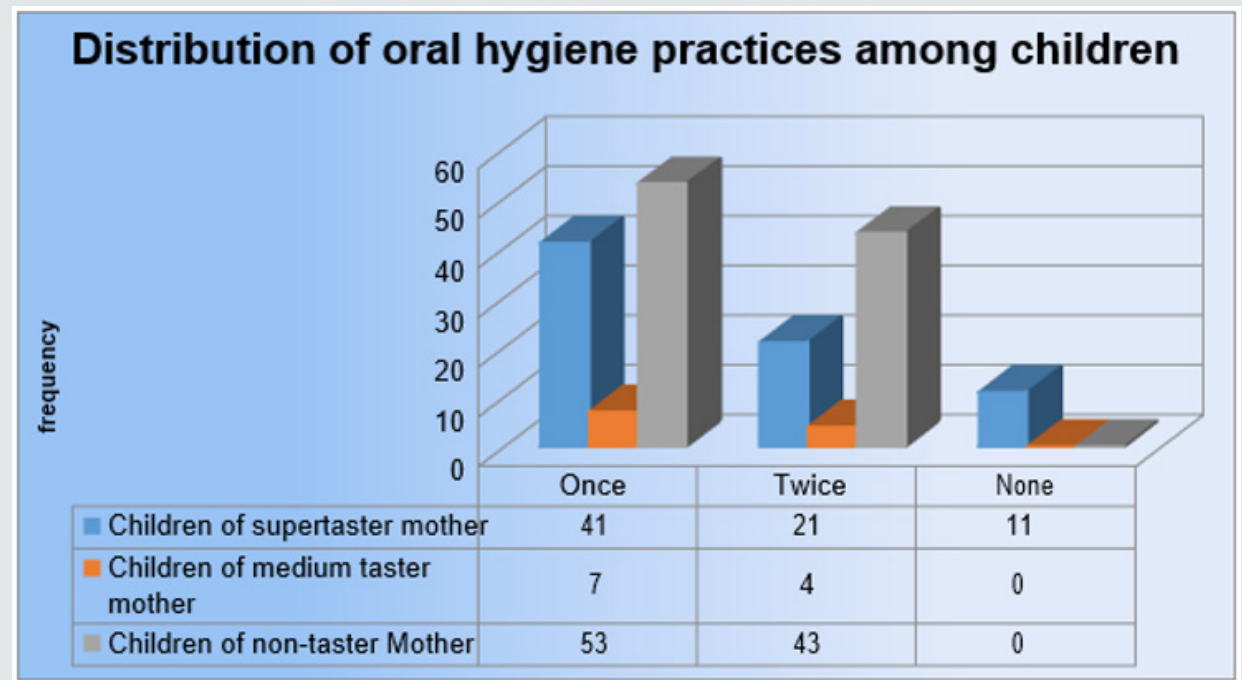

Figure 5: Distribution of oral hygiene practices among children.

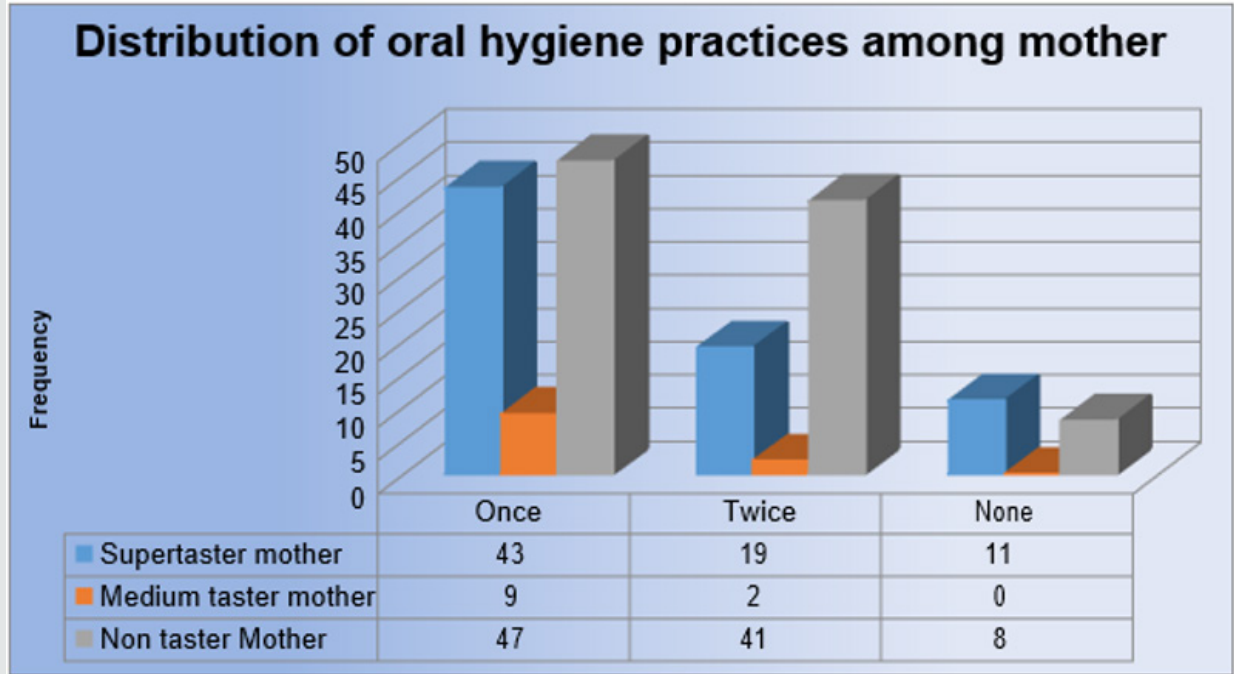

Figure 6: Distribution of oral hygiene practices among mother.

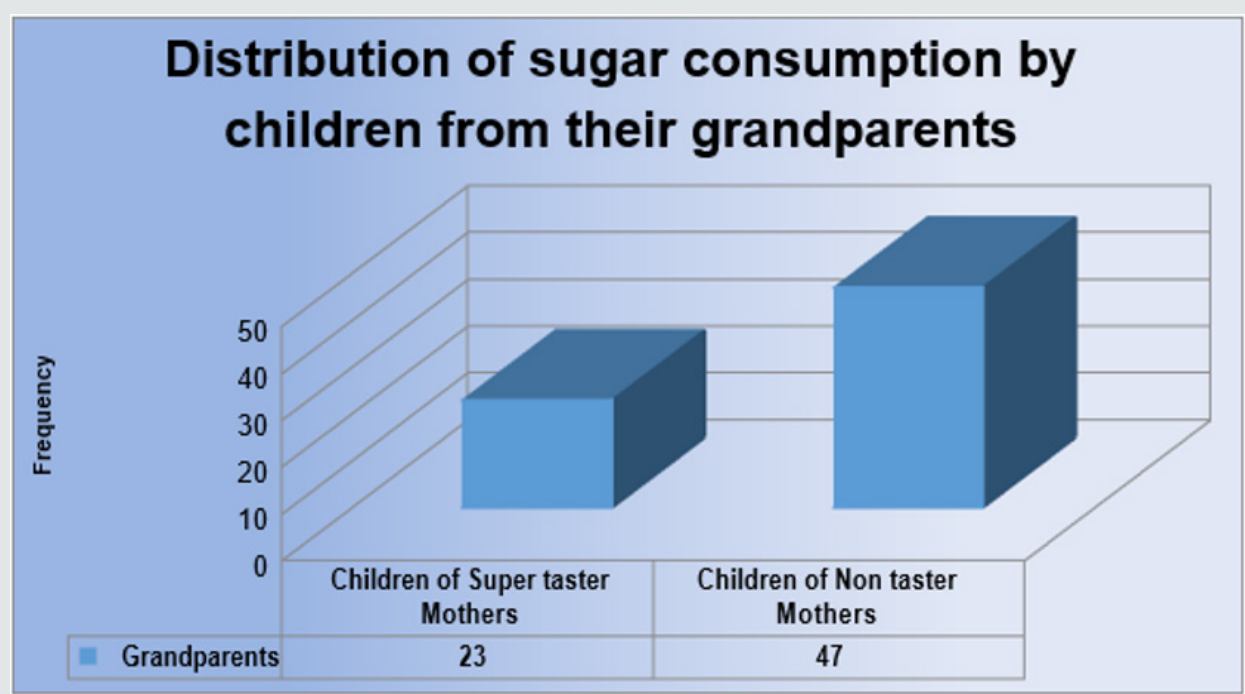

Figure 7: Distribution of sugar consumption by children from their grandparents. 


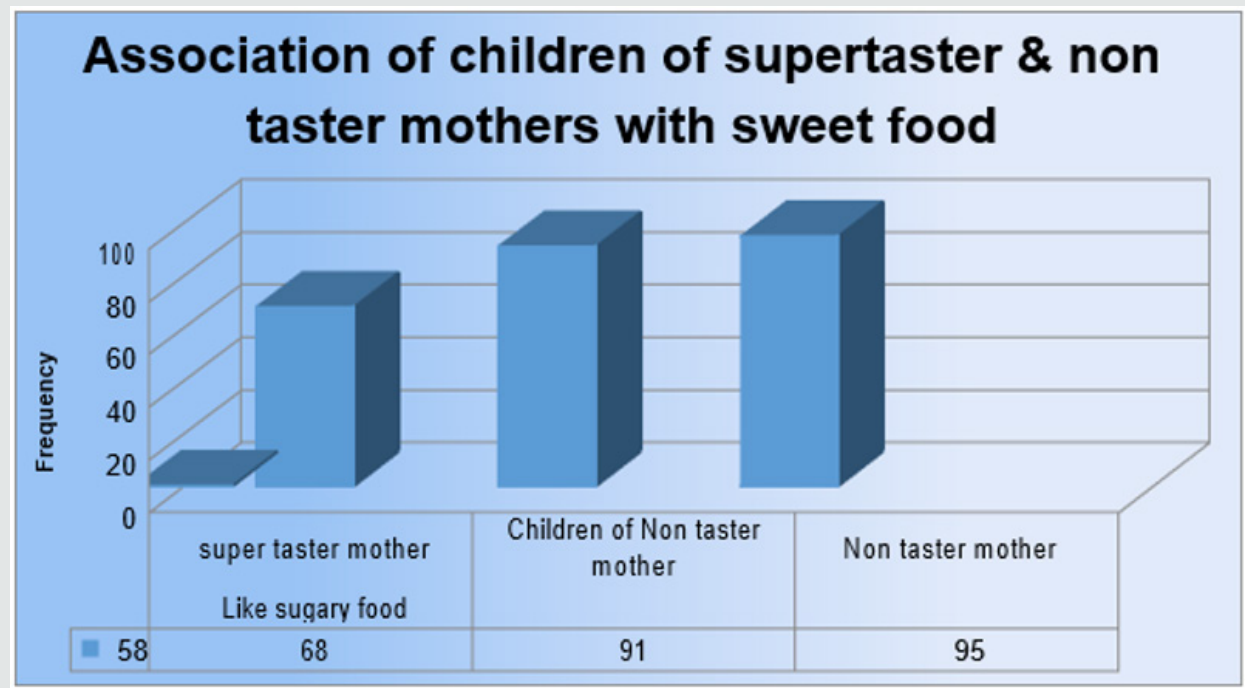

Figure 8: Association of children of supertaster \& non taster mothers with sweet food.

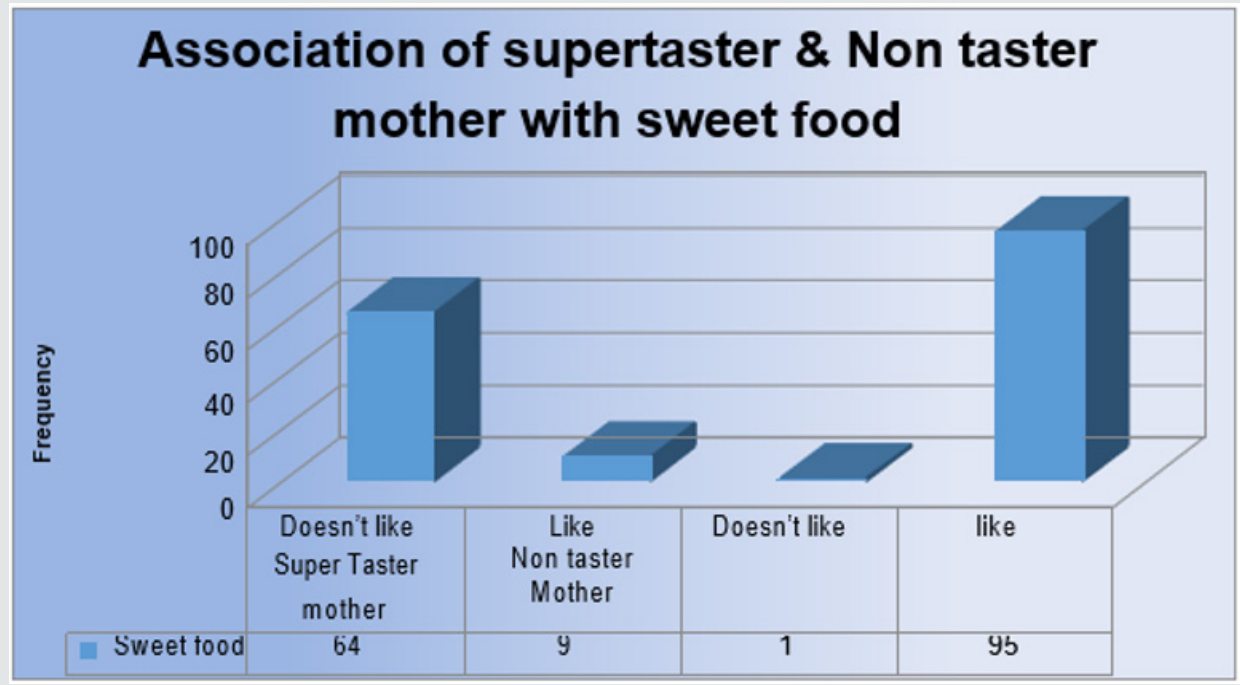

Figure 9: Association of supertaster \& Non taster mother with sweet food.

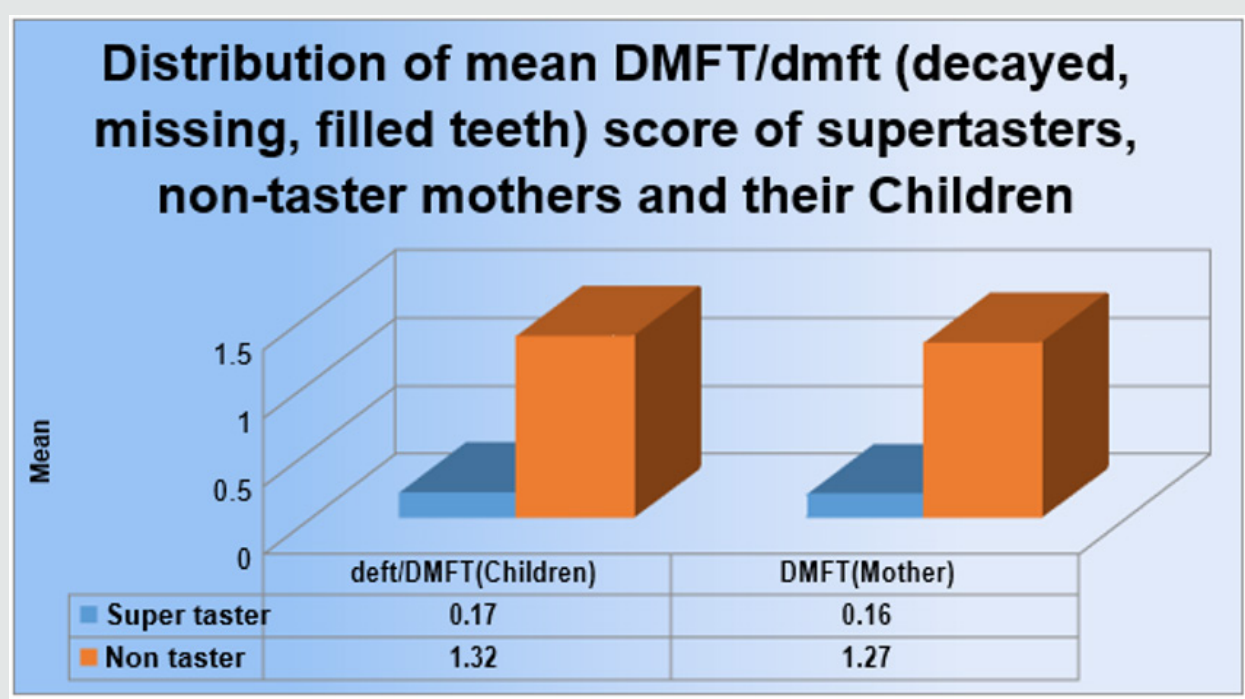

Figure 10: Distribution of mean DMFT/dmft score of supertasters, non-taster mothers and their Children. 
Table 1: Association of supertaster \& non taster mothers and their children with sweet food.

\begin{tabular}{|c|c|c|c|c|}
\hline \multirow{2}{*}{} & \multicolumn{2}{|c|}{ Doesn't like sugary food } & \multicolumn{2}{c|}{ Like sugary food } \\
\cline { 2 - 4 } & $\begin{array}{c}\text { Children of supertaster } \\
\text { mother }\end{array}$ & Super taster mother & Children of Non taster & Non taster mother \\
\hline Sweet food & $58(79.4 \%)$ & $68(87.6 \%)$ & $91(94.7 \%)$ & $95(98.9 \%)$ \\
\hline Unpaired t-test & $0.01^{* *}$ & & $0.01^{* *}$ & \\
\hline
\end{tabular}

Table 2: Distribution of mean DMFT/dmft score of supertasters, non-taster mothers and their Children.

\begin{tabular}{|c|c|c|}
\hline & dmft/DMFT(Children) & DMFT(Mother) \\
\hline Super taster & 0.17 & 0.16 \\
\hline Non taster & 1.32 & 1.27 \\
\hline
\end{tabular}

\section{Discussion}

The present study comprised of 180 pairs of mother and child. The mothers were subjected to PROP testing and classified into super tasters, medium tasters and non-tasters according to the Green's labeled magnitude scale. The mothers who were super tasters found the taste of PROP to be extremely bitter and the non-taster mothers described the taste of PROP to be tasteless while the medium taster mothers failed to categories the taste of PROP. The super taster mothers perceived the taste of PROP in lower concentration (1.6 $\mathrm{mg}$ per $\mathrm{ml}$ ). This may be due to the presence of high-density fungiform papilla and taste receptors in the tongue compared to medium tasters and non-tasters. This may make the super taster mothers dislike sugary food. Mothers being the primary care givers to children influences the food habit of children. Mothers who prefers sugary foods are more likely to prepare and feed their children the same then those who do no prefer sugary food. In our study it was found that the mean dmft/ DMFT score among non-taster mothers (1.32) and their children (1.27) was higher than the mean dmft/ DMFT of super taster mothers (0.17) and their children (0.16) respectively. The influence of grandparents in the family cannot be neglected. Even in children of super taster mothers, 23 children reported they were given sugary snacks by their grandparents regularly while 47 children of non-taster mothers reported the same.

The pampering of grand parents may also be a causative factor for dental caries in children. The finding of the present study also showed that children who brushed their teeth once or twice per day experienced more dental caries if their mothers were non tasters whereas children of super tasters had less dental caries. This can be explained by the frequent intake of sugary food in case of children of non-taster mothers and also may be influenced by the food prepared by their mothers. Similarly, mothers who were non tasters had a higher dental caries experience than the super taster mothers. This may be due to their increased susceptibility to sugary food. In the present study when the super tasters and non-taster mothers were asked about their liking for sugary food, majority of the super taster mothers (64) stated that they did not prefer sweet food, while 95 non taster mothers stated that they preferred sugary food. In case of the children of super taster and non-taster mothers, the results were significant. The mother's dietary habits and tastes probably affected the oral health status of the child. When the association between the preference of sugary food in super taster and non-taster mothers and their children was seen, it was found that the children of super taster mothers did not prefer sugary food while it was vice versa for the children of non-taster mothers. This may be due to genetic factors and also might be due to the dietary habits of the mothers which influenced the dental caries status of their children.

\section{Conclusion}

In the present study, the results suggested that the children of the mothers who are non-tasters or who prefers sweet food have higher dental caries experience. Factors such as the presence of grandparents in the household, frequency of brushing, preference of food and dietary habits may play an important role in the development of dental caries in children. Thus, mother's genetic sensitivity to the bitter taste of prop can be used as a useful adjunct to identify children who are at risk of developing dental caries.

\section{References}

1. Rupesh S, Nayak UA (2006) Genetic sensitivity to the bitter taste of 6-n-propylthiouracil, A new risk determinant for dental caries in children. J Indian Soc Pedod Prevent Dentist 24(2): 63-68.

2. Seow WK (1998) Biological mechanism of early childhood caries. Commun Dentist Oral Epidemiol 26(Suppl 1): 8-27.

3. Anil S, Anand PS (2017) Early Childhood Caries: Prevalence, Risk Factors, and Prevention. Front Pediatr 5: 157.

4. Mazengo MC, Tenovuo J, Hansen H (1996) Dental Caries in relation to diet, saliva and cariogenic microorganism in Tanzanians of selected age groups. Common Dentist Oral Epidemiol 24(3): 169-174.

5. Johnson NW (1991) Introduction: The nature of the caries process and the need for markers at risk. In: Johnson NW (editor) Risk marker for oral diseases-volume 1- Dental caries $1^{\text {st }}$ ed. Cambridge University Press, Cambridge, USA. p. 1-12.

6. Vargas CM, Crall JJ, Schneider DA (1998) Sociodemographic distribution of pediatric dental caries: NHANES III, 1988-1994. J Am Dent Assoc 129(9): 1229-1238.

7. Vandal VB, Noorani H, Shivaprakash P K, Walikar B, (2017). Genetic specificity to 6-n-propylthiouracil and its association to dental caries: A Comparative study. J Indian Soc Pedod Prev Dent 35(1): 83-85.

8. Hicks J, Garcia Godoy F, Flaitz C, (2003) Biological factors in dental caries: Role of saliva and dental plaque in the dynamic process of 
demineralization and remineralization (part 1). J Clin Pediat Dentist 28(1): 47-52.

9. Lehl G, Bansal K, Sekham R (1999) Realtionship between cariogenic diet and dental caries as evaluated from a 5- day diet diary in 4-12 years old children. J Indian Soc Pedod Prevent Dentist 17: 119-121.

10. Beighton D, Adamson A, Rugg Gunn A, (1996) Association between dietry intake, dental caries experience and salivary bacterial levels in 12-year-old English school children. Arch Oral Biol 41(3): 271-280.
11. Anliker JA, Bartoshuk L, Ferris AM, Hooks LD (1991) Children's food preferences and genetic sensitivity to the bitter taste of 6-n-propylthiouracil (PROP). Am J Clin Nutr 54(2): 316-320.

12. Bartoshuk LM, Duffy VB, Lucchina LA, Prutkin J, Fast K (1998) PROP (6-n-propylthiouracil) supertaster and the saltness of $\mathrm{NaCl}$. Annal New York Acad Sci 855: 793-796.

\section{(C) \\ This work is licensed under Creative Commons Attribution 4.0 License}

To Submit Your Article Click Here:

Submit Article

DOI: $10.32474 /$ IPDOAJ.2020.03.000168

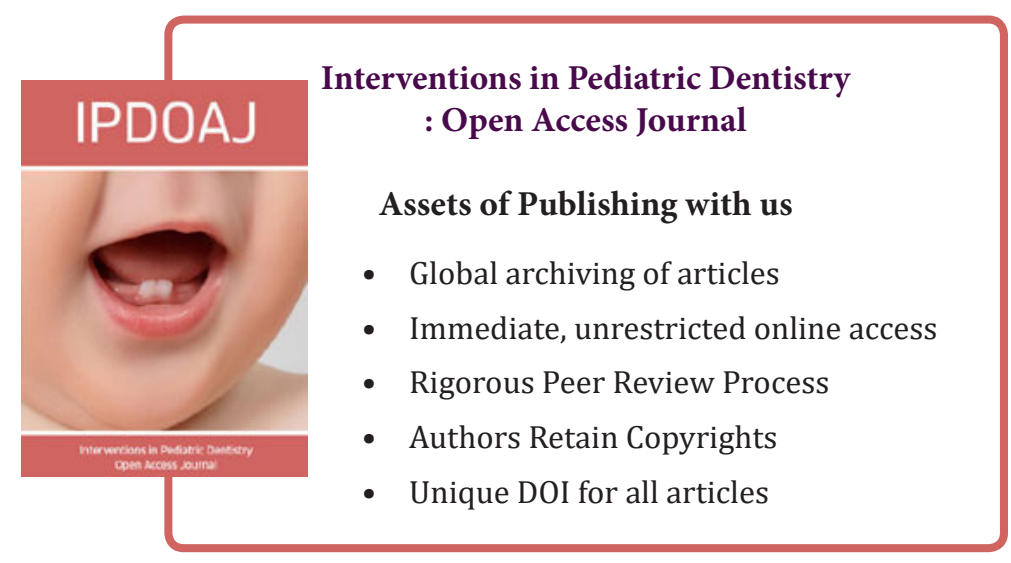

